\title{
Article \\ Localisation of Room Occupants in Home Environments using Unobtrusive Sensing Solutions
}

\author{
Idongesit Ekerete ${ }^{1}$, Chris Nugent ${ }^{1}$ and Jim McLaughlin ${ }^{2 *}$ \\ 1 School of Computing, Jordanstown Campus, Ulster University, United Kingdom. \\ 2 School of Engineering (NIBEC), Jordanstown Campus, Ulster University, United Kingdom. \\ * Correspondence: ekerete-i@ulster.ac.uk; Tel.:+447438315653
}

\begin{abstract}
This paper proposes the localisation of room occupants in home environments using Unobtrusive Sensing Solutions (USSs). The ability to localise room occupants in home environments can help in the objective monitoring of sedentary behaviour, control of appliances and temperature regulation inside buildings. While wearable sensors can provide tangible information on health and wellness, they have battery-life issues and the inability to perform prolonged monitoring. This work uses heterogeneous USSs in the form of an Infrared Thermopile Array (ITA-64) thermal sensor and a Multi-Chirp Frequency Modulated Continuous Wave Mono-pulse (MC-FMCW-M) Radar sensor to monitor room occupants. Digital filters, interpolation and background subtraction algorithms were used to process the thermal images gleaned from the ITA-64 thermal sensors. The MC-FMCWM Radar sensor used multi-chirp and Doppler shift principles to estimate the exact location of the targeted room occupants. The estimated distances from the Radar sensor were compared with ground-truth values. Experimental results demonstrated the ability to identify thermal blobs of occupants present in the room at any particular time. Data analyses indicated no significant difference $(p=0.975)$ and a very strong positive correlation $(r=0.998)$ between the ground-truth distance values and those obtained from the Radar sensor.
\end{abstract}

Keywords: Unobtrusive Sensing; Radar sensor; Thermal Sensor; Localisation; Home Environment.

\section{Introduction}

The history of wearable devices dates to the 15th century with the advent of simple wristwatches to tell time [1]. In the past five decades, additional functions have been integrated into Wearable Sensing Solutions. These add-ons have included health monitoring [2], [3] and activity recognition functions [4]-[6], amongst others. While emphasising the numerous advantages of using wearable devices for monitoring, it is worth highlighting that some of the devices are designed with unpleasant entanglements. Others are recognised for having battery-life and wearability issues. As an example, a study by [3] on localisation for activity recognition required participants to wear sensors on seven different parts of the body. With these numerous sensors and entanglements, users can be discouraged from wearing and continual usage of the proposed technology. Also, uninterrupted usage of wearable devices for localisation in a home environment can be impracticable, uncomfortable or undesirable due to wearability issues and other issues such as muscle artefacts, which can affect the quality of data obtained from the devices. In addition, the ability of users to remember to charge and wear devices can result in frequent interruptions of data acquisition processes.

\section{Related Work}

Unobtrusive Sensing Solutions (USSs) are being used increasingly for the purposes of indoor and outdoor localisation. They include monitoring devices that are not worn or held on any part of the human body. Recently, these devices have been applied in many 
health-related and other indoor settings, such as monitoring at-risk individuals [7], the performance of Activity of Daily Living (ADLs) [8], exercise rehabilitation [9] and localisation frameworks [10], [11]. Whilst some of these sensing solutions can work independently, others need to be integrated with wearable solutions.

The unmanned aerial vehicle is an example of an outdoor system for manoeuvring rugged terrains in the security and surveillance framework. Another example is the global positioning system (GPS) which can be used to collect data for location estimation and mapping. Incident localisation and identification such as earthquakes, highway congestions, environmental pollution, missiles and drones invasion can also be carried out with the help of the GPS, global navigation satellite system and other related IoT technologies [12]-[14]. Unfortunately, GPS is not suitable for indoor data acquisition in places such as underground buildings, railway stations and parking garages [15].

An indoor positioning system (IPS) includes a network of sensors that can locate objects or people inside buildings and confined spaces. In order to evaluate the performance of an IPS, some metrics such as accuracy, responsiveness, cost, range and adaptiveness to the environment [16] are often considered. IPS such as Infrared and Radiofrequency (RF) based solutions can be used to locate people and objects indoors [17]. RF technology can be found in a wide range of devices and applications. These include ZigBee, Bluetooth, WiFi antennas, Radar and RF Identification (RFID) devices [16]. Although they have made significant contributions as IPSs, a review of their performance is necessary for future improvement.

RFID systems have been widely used in IPS. These systems use radio waves to identify and track their targets. The tracking information is stored on tags placed on intended targets. RFID systems are used in many industries, such as the automobile manufacturing industries [18], to track the progress and location of items along assembly lines. At security checkpoints, RFID tags are used in many locations as a replacement for magnetic stripe cards. Nevertheless, passive tags suffer from received-signal-strength-indication loss. Furthermore, active RFID tags suffer from battery-life challenges [18]. Although efforts have been made to reduce these problems through the use of advanced RFID technologies, limitations, such as single object tracking, direction dependence and non-conformity with the Federal Communications Commission power limits standard [19], are still being researched.

Spot localisation has also been explored using information from the physical layer in WiFi systems. Sen et al. [20] used channel responses in a WiFi network as a location signature to obtain location information of users of the network. This study, which attained a mean accuracy of $89 \%$ in the localisation of users, however, had issues with the mobility of users, dependence on a hardware card and device orientation. Also, the study required all participants to be connected to a WiFi network. Hence, unconnected users failed to be localised. Another WiFi-based research conducted by Wang et al. [21] for gait recognition suffered a similar challenge. The challenge included having users walk in a predefined direction which fell short of real-life experience.

Bluetooth uses short-wavelength ultra-high-frequency radio waves and a personal area network for data exchange. It operates around $2.4 \mathrm{GHz}$ industrial scientific and 
medical radio band [16]. For Bluetooth communication to be established, communicating devices need to be paired and within a line of sight. Although Bluetooth technology is advantageous as an IPS because of its low cost, high security, lower power and small size, it however, requires users to always remember to turn on the Bluetooth App. Another drawback is localisation lags experienced in device discovery and authentication procedures [16].

Similar to Bluetooth, ZigBee is an IEEE 802.15 based wireless technology that enhances medium and short-range communications. It is an ideal communication tool for low-power and small data exchange. A ZigBee network uses reference sensors with known locations to determine the location of an unknown sensor normally referred to as the blind sensor. Fang et al. [22] presented a framework for ZigBee IPS by integrating different algorithms during a localisation procedure. The framework estimated locations with more accuracy when compared with other ZigBee frameworks. The framework was not, however, compared with the research outcomes from other IPSs such as Infrared or Radar technology.

The Ultra-Wide-Band (UWB) Radar is a high bandwidth radio technology system that utilises low energy for short-range communications. Access points and algorithms for UWB Radar during an indoor localisation study was proposed by [23]. Mobile units (MUs) were used as targets for this study. Although a high accuracy was reported in the resulting overall measurements, the study setup posed challenges to home-based users. Also, MUs were deployed in the study instead of human targets.

This study, therefore, proposes the localisation of room occupants in home environments using USSs such as ITA-64 thermal sensor and an MC-FMCW-M Radar sensor. The main contribution of this work are itemised as follows: (i) to determine the actual location of room occupants in home environments using heterogeneous USSs, (ii) to demonstrate the advantages of using USSs such as Radar and Thermal SS in indoor monitoring against the wearable solutions, (iii) to demonstrate the advantages of using dual and complementary monitoring solutions compared with using a single SS, (iv) to monitor human presence in a room through the usage of USSs such as thermal and Radar SS.

\section{Materials and Methods}

The experimental setting comprised a Silicon-Germanium ( $\mathrm{SiGe}$ ) based $24 \mathrm{GHz}$ Multi-Chirp Frequency Modulated Continuous Wave Mono-pulse (MC-FMCW-M) Radar chipset and a-80-by-64 resolution Infrared Thermopile Array (ITA-64) sensor. While the Radar module targeted range values (distances) of room occupants, the ITA-64 sensor was utilised for their thermal blobs. Both sensors were placed in a 3D-printed enclosure and mounted on a tripod stand, as shown in Figure 1. 


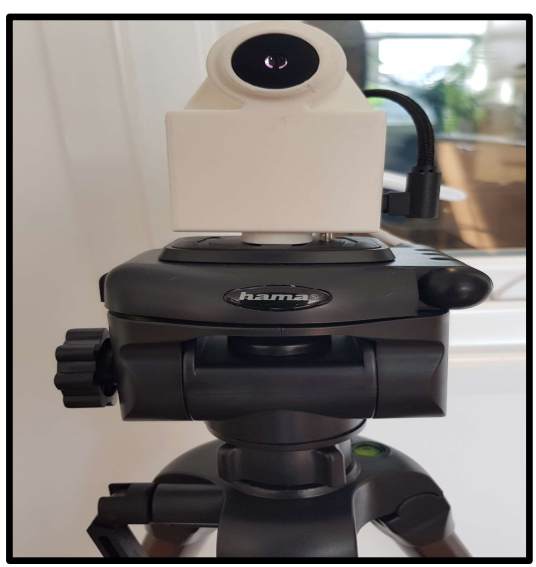

Figure 1. Thermal and Radar Sensors placed in a 3D enclosure and mounted on a tripod stand during data collection.

The office space in which the study was conducted is presented in Figure 2. The rationale for considering heterogeneous USSs such as the ITA-64 thermal sensor and the MC-FMCW-M Radar sensor in this study was for complementary monitoring. Others included accuracy improvement and the obtainment of privacy-friendly postures of room occupants.
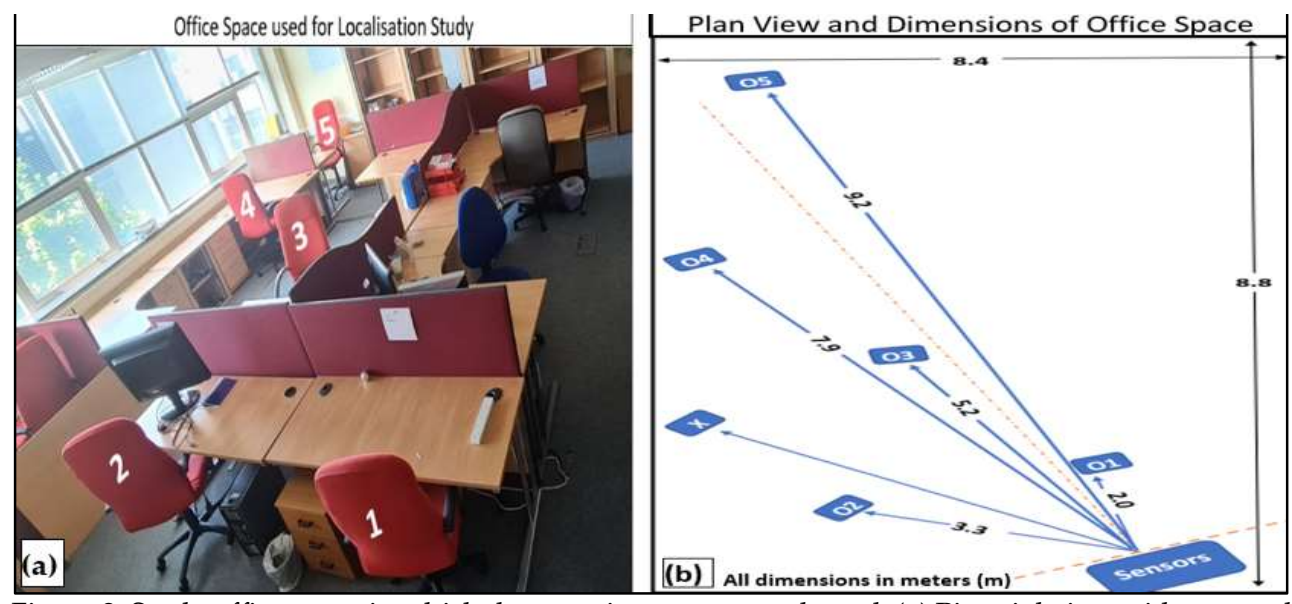

Figure 2. Study office space in which the experiment was conducted. (a) Pictorial view with targeted room occupants numbered $1-5$, (b) The plan view and dimensions of the office space used for this study. All dimensions are in meters $(m)$. Figure $2(b)$ is not drawn to scale.

The pictorial view of the sitting arrangement of the targeted room occupants is presented in Figure 2. The targeted room occupants referred to as O1, O2, O3, O4 and O5 (in this study) were at seats number 1, 2, 3, 4 and 5, respectively. In Figure 2(b), the plan view of the sitting arrangement, $\mathrm{O} 1$ sat very close to the sensors at 2.0 meters, $\mathrm{O} 5$ was farther away at 9.2 meters. O2, O3, and $\mathrm{O} 4$ were 3.3, 5.2, 7.9 meters from the sensors, respectively. The office space measures $8.8 \mathrm{~m}$ by $8.4 \mathrm{~m}$ by $2.7 \mathrm{~m}$ for length, width and height, respectively. It has 13 spaces, out of which 5 regularly occupied spaces were targeted for the study.

Given that the sensors were mounted on a tripod stand $1.7 \mathrm{~m}$ above ground level, there was a difference in height between the average height of the occupants in a sitting position and the height of the sensor. The average of the 5 occupants' heights (on their seats) was calculated to be $1.3 \mathrm{~m}$; their respective distances from the sensors were then computed using the Pythagoras theorem. Figure 3 presents a view of the difference in 
heights and the position of the SSs. Signal propagation from the SSs to the occupants is represented by the hypotenuses (in red, Figure 3).

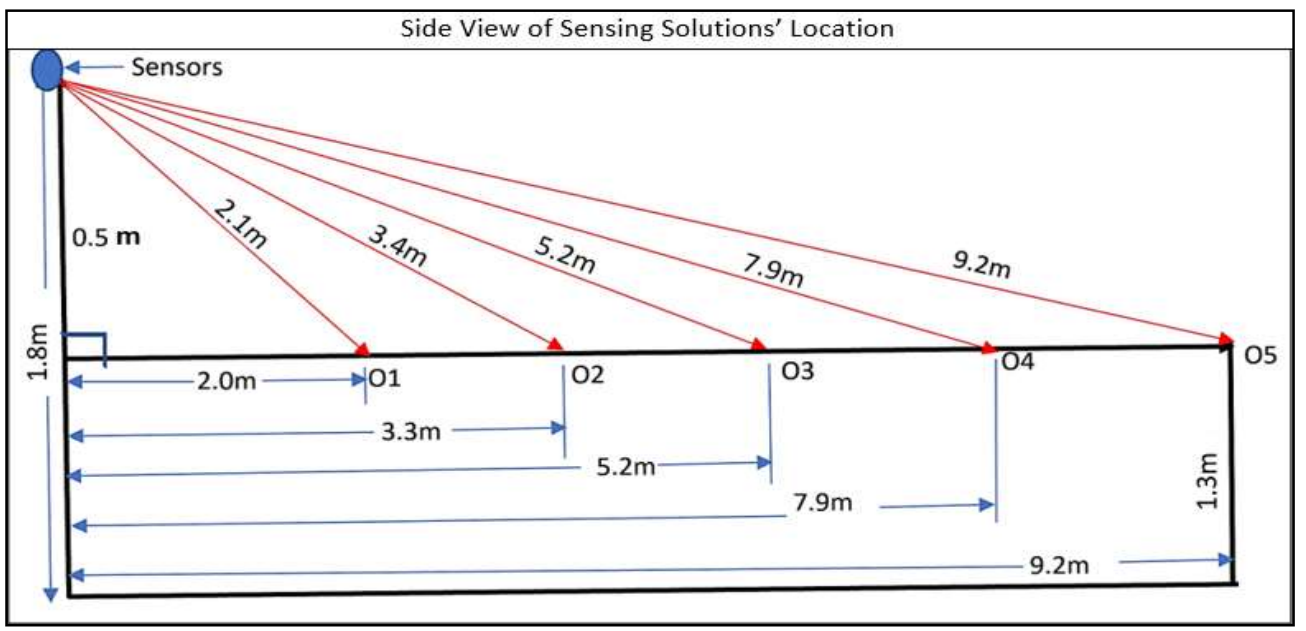

Figure 3. Side view of sensors location, height and measured distances of targeted occupants designated as $\mathrm{O} 1$ to $\mathrm{O} 5$.

The Radar sensor was used to record live data from the targeted occupants for ten days. The sensor was interfaced with MATLAB software. The ITA thermal sensor recorded the thermal blobs of all the occupants simultaneously to allow for easy playback. It is important to emphasise that the participants' daily activities and office duties were neither influenced nor interrupted in any way during the data acquisition process. Also, their sitting positions were not adjusted either, and data collection continued without any adjustment to their daily routines.

\section{Results}

Data obtained from the MC-FMCW-M Radar sensor was analysed using MATLAB 2018 and Minitab 18 statistical software packages. Range values gleaned from the sensor were checked for zero-factor error (ZFE) before being exported to Minitab for descriptive analysis. A total of 565 frames were considered for descriptive analysis. The mean, standard error of the mean (SEM) and standard deviation (SD) of the range values were computed for each occupant as presented in Table 1. Other parameters considered were the variance and the coefficient of variance (CoefVar) of each of the range values. Thermal blobs gleaned from the ITA sensor were binarised to improve their granularity. A digital image interpolation algorithm was used to eliminate thermal blobs interference.

Table 1: Descriptive statistics of MC-FMCW-M Radar range values for O1 to O5 representing the 5 occupants that participated in the study.

\begin{tabular}{|l|l|l|l|l|l|l|}
\hline \multicolumn{7}{|c|}{ Descriptive Statistics of Range Values of Targeted Room Occupants } \\
\hline $\begin{array}{l}\text { Varia- } \\
\text { ble }\end{array}$ & $\begin{array}{l}\text { Total count of range } \\
\text { values per occupant }\end{array}$ & Mean & SE Mean & St. Dev. & $\begin{array}{l}\text { Vari- } \\
\text { ance }\end{array}$ & $\begin{array}{l}\text { Coef. } \\
\text { Var. }\end{array}$ \\
\hline O1 & 113.0 & 2.1 & 0.0 & 0.1 & 0.0 & 5.2 \\
\hline O2 & 113.0 & 3.3 & 0.0 & 0.1 & 0.0 & 2.8 \\
\hline O3 & 113.0 & 5.3 & 0.0 & 0.0 & 0.0 & 0.9 \\
\hline O4 & 113.0 & 7.9 & 0.0 & 0.1 & 0.0 & 0.8 \\
\hline
\end{tabular}




\begin{tabular}{|l|l|l|l|l|l|l|}
\hline O5 & 113.0 & 8.8 & 0.0 & 0.2 & 0.0 & 1.8 \\
\hline
\end{tabular}
Legend: SE Mean = Standard Error of the Mean. St Dev = Standard Deviation. Coef. Var. = Coef-
ficient of Variance

Results presented in Table 1 indicated a SD of 0.3 for all five targeted occupants. This implies that the 113 range values for each occupant were within a close boundary. Participant O5, however, had the highest value of SEM value compared with other occupants due to their distance from the sensor. This is followed by $\mathrm{O} 1$ because of two noticeable factors. Firstly, the height of the sensor, which created an offset on the target because of sitting too close to the sensor and secondly, frequent adjustment of sitting positions during data acquisition. These factors resulted in a higher value of CoefVar (5.2) in O1 compared with the other targeted occupants. The value was, however, smaller when compared with other acceptable standard values [24].

A 2-sample T-test of the mean range values for the Radar SS and the ground-truth values (from a meter rule) at a 95\% confidence interval indicated that there was no significant difference $(\mathrm{p}=0.975)$ between them. Also, a T-value of 0.03 at 7 degrees of freedom showed a no-departure of the estimated Radar sensor's range values from the groundtruth measurements. A Matrix plot with correlation coefficient is presented in Figure 4. The $x$ and $y$ axes on the plot represent the mean Radar sensor and the ground-truth range values for each occupant.

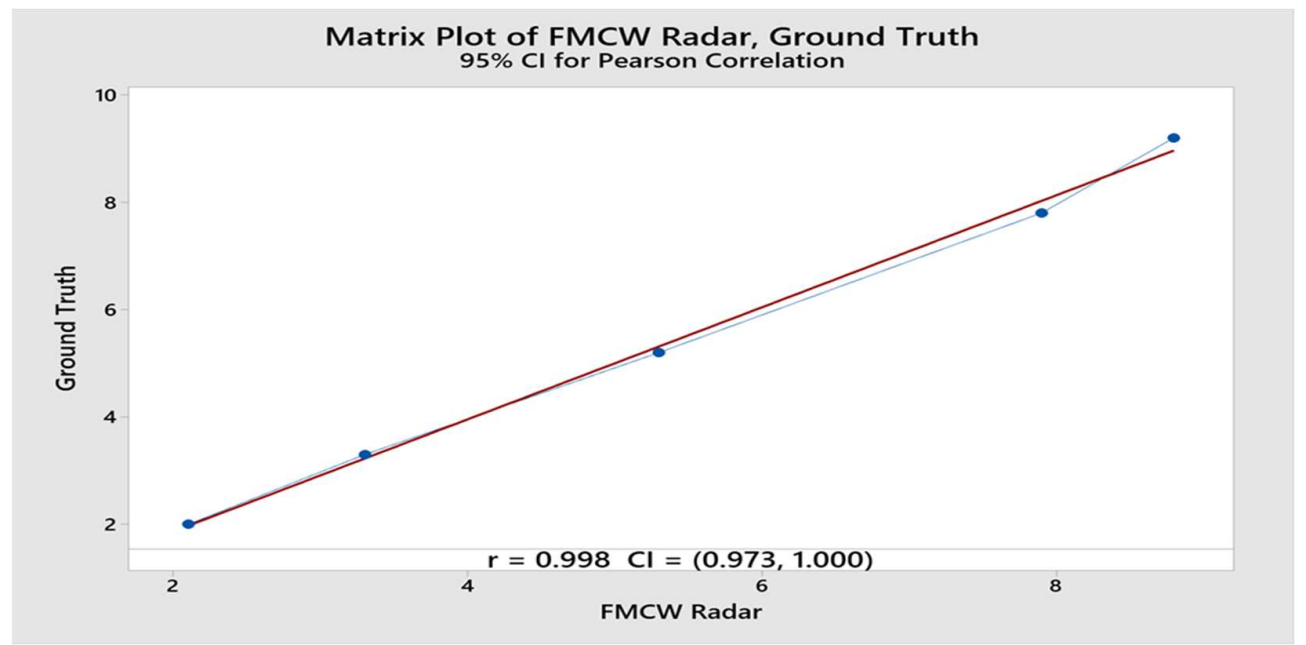

Figure 4. Matrix plot of the mean MC-FMCW-M and ground-truth range values at 95\% CI for Pearson correlation.

The direction and strength of the linear relationship between the two sets of range values is measured by the Pearson correlation coefficient, r. Figure 4 indicates a strong positive relationship between the ground-truth and the Radar sensor's range values $(r=$ 0.998). These results further underscore the high accuracy and reliability of the Radar estimated range values in this study.

The thermal blobs of the room occupants were captured with the ITA sensor when all the occupants were at their respective seats. This was also the case with the MCFMCW-M Radar. Unlike other studies [18] [19] that restricted participants to a predetermined pattern, the occupants (in this study) were not advised to conform to any specific pattern during data collection. This approach was taken to allow for real-life behaviour 
rather than experimentally constrained behaviour, which may influence experimental outcomes. Figure 5(a) and (b) present the ITA actual and binarised thermal blobs, respectively of the targeted room occupants.

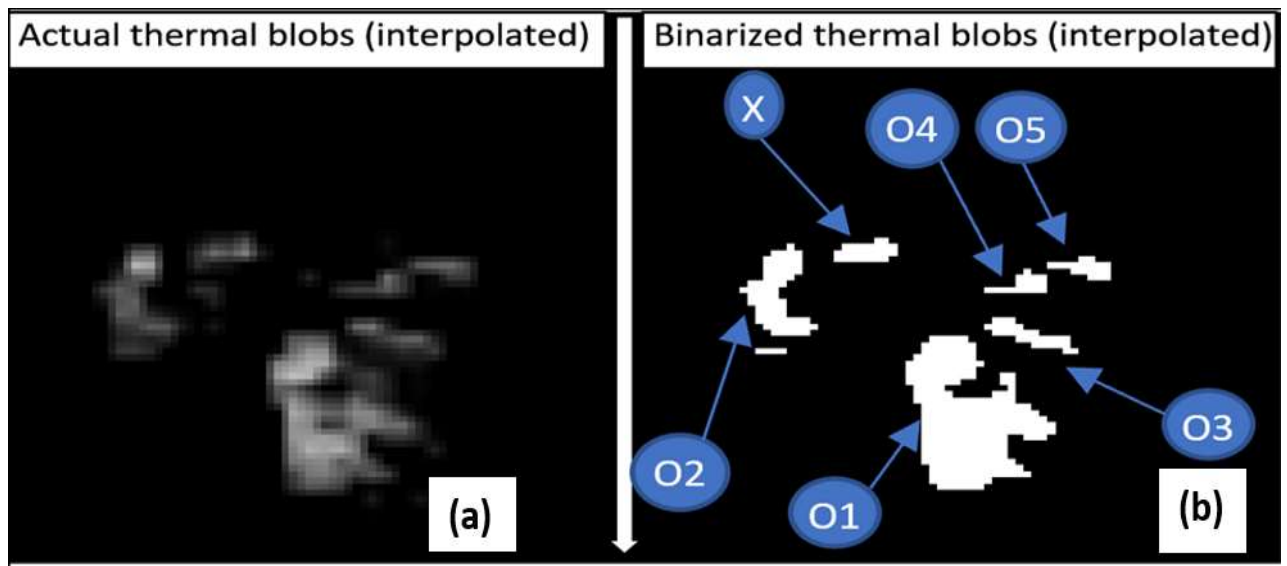

Figure 5. ITA-64 Thermal blobs of targeted room occupants. (a) actual blobs (b) binarised blobs.

Acquired thermal blobs were binarised (Figure 5(b)) to enhance clarity, more so, to represent a distinct thermal blob of each targeted participant. From Figure 5(b), O1 and $\mathrm{O} 2$ had almost a third of a full human thermal blob due to their proximity to the sensor. Thermal blobs from O3, O4 and O5 appeared smaller; however, they were still visible and distinguishable. The occupant marked ' $X$ ' was not included in the study. Additionally, it was necessary to eliminate heat signatures from computers and other heat-generating devices using background subtraction algorithms. Also, enabling thermal filters such as Median, FIR, and framestack reduced image bickering, thus the smooth thermal blobs in Figure 5(a) and (b).

Moreover, the digital image interpolation (DII) algorithm played an important role in distinguishing each occupant's thermal blobs during data processing. It also helped in eliminating interference from the thermal blobs. In this study, the DII algorithm was utilised to shrink thermal blobs from one-pixel orientation to the other. This became necessary to avoid pixel scattering and interference. Figure 6(a) and (b) present both the actual and binarised thermal blobs without interpolation, respectively.

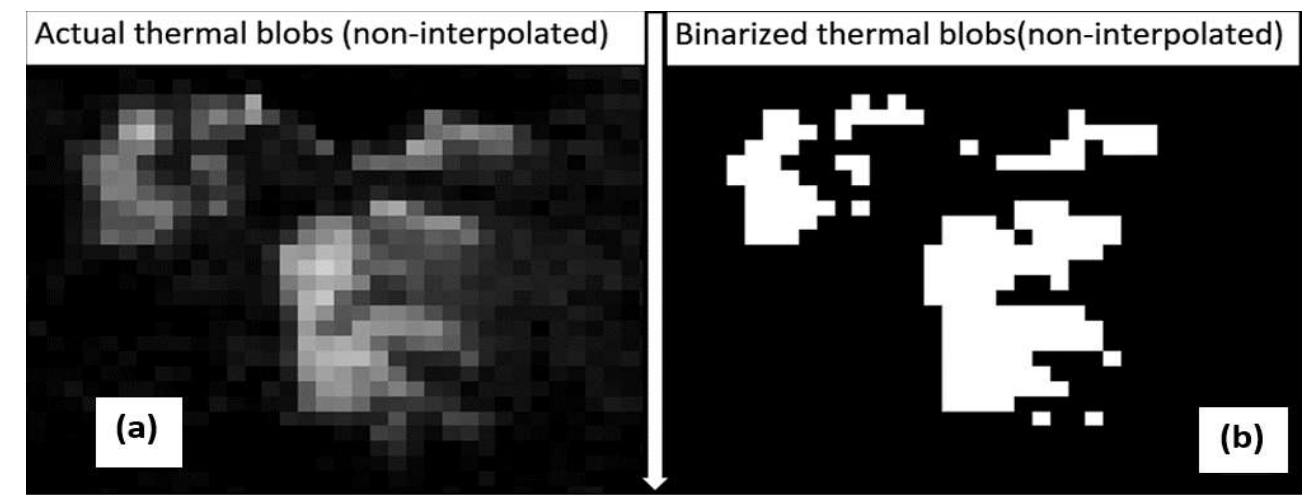

Figure 6. ITA-64 Thermal Blobs of room occupants without interpolation. (a) Actual (b) Binarised.

In Figure 6, the thermal blobs of $\mathrm{O} 1$ and $\mathrm{O} 3, \mathrm{O} 5$ and $\mathrm{X}$ interfered with one another.

Also, loose pixels are observed, which can be mistaken for additional human thermal 
blobs. Comparing Figure 5 and Figure 6, the advantage of deploying the DII algorithm is well demonstrated. Nevertheless, although visible and clear thermal blobs were obtained for each targeted occupant in the room, it was difficult to estimate the actual distances with the thermal sensor. Hence, the Radar sensor complemented this function by acquiring distances of the targeted room occupant. Thus, combining information from the Radar and the thermal SSs, it was easier to estimate each room occupant's actual location and detect their presence and absence in the room.

\section{Discussion}

The experimental evidence from this study has informed the benefits of using USSs such as MC-FMCW-M Radar and ITA-64 thermal SSs for IPS. Hence, the proposed USSs in this study can be considered as better alternatives to existing IPS such as GPS, RFID, Bluetooth, ZigBee and WiFi antennas. Promising results from this study through the usage of heterogeneous USSs can help curb cases of false positives, which was highlighted by [25] as one of the prevalent issues in existing technologies. With dual and complementary USSs such as those used in this study, the false positives that are usually associated with single sensor monitoring would be eliminated. Another advantage of this study includes monitoring multiple occupants at the same time. The MC-FMCW-M Radar and ITA sensors are able to monitor multiple room occupants at the same time. These added advantages, including their non-obtrusive characteristics, make them preferred to wearable solutions customarily required to be worn by each room occupant.

This study has many areas of practical application, including monitoring ageing adults in a home environment, multiple occupants in a care facility, sedentary behaviour in a workspace [26] and temperature control in offices and homes, to name but a few. Although diverse smart SSs are currently used for these purposes, a recent systematic survey by ElHady and Provost [25] detailed that more work was still required in terms of addressing failure and faults in the existing systems.

The main challenge with this study was the ZFE. It occurred under two circumstances in this study. Firstly, when a reflected signal from a target is not received at the sensor. Secondly, when an occupant left their seat during the experiment. While the first instance has a negative influence on the data recorded by the sensor, the second instance did not affect the recorded data. In order to eliminate the first case, data were manually cleaned with the help of complementary information from the ITA sensor. This further highlights the essence/benefits of heterogeneous sensing considered within this study.

\section{Conclusions}

This study presented privacy-friendly heterogeneous USSs for localisation of room occupants in a home environment. An MC-FMCW-M Radar and an ITA SSs were considered. Each SS was non-wearable, contactless and unobtrusive. The sensors individually acquired data and processed the data for complementary functions. Data acquisition was carried out for ten days, and a total of 565 data frames were analysed from both sensors. Experimental results clearly indicated that the Radar sensor estimated the range values of the targeted participants with high accuracy. The ITA sensor complementarily recorded distinct thermal blobs, which, in combination with the Radar sensor data, estimated the actual sitting location of the targeted occupants. These traditional roles helped to avoid 
zero-factor and false-positive errors. Future work will attempt the use of these USSs alongside data mining algorithms to localise and predict the speed at which ADLs are performed in a home environment.

Funding: Research is funded by the EU's INTERREG VA program, managed by the Special EU Program Body (SEUPB).

\section{References}

[1] C. Bishop, Pattern Recognition and machine Learning. Springer New York LLC, 2006.

[2] C. Vu and J. Kim, “Muscle activity monitoring with fabric stretch sensors," Fibers Polym., vol. 18, no. 10, pp. 1931-1937, Oct. 2017, doi: 10.1007/s12221-017-7042-x.

[3] L. Gao, A. K. Bourke, and J. Nelson, “Evaluation of accelerometer based multi-sensor versus single-sensor activity recognition systems," Med. Eng. Phys., 2014, doi: 10.1016/j.medengphy.2014.02.012.

[4] B. Kwolek and M. Kepski, "Improving fall detection by the use of depth sensor and accelerometer," Neurocomputing, vol. 168, pp. 637-645, Nov. 2015, doi: 10.1016/j.neucom.2015.05.061.

[5] M. Garcia-Constantino, A. Konios, M. A. Mustafa, C. Nugent, and G. Morrison, “Ambient and Wearable Sensor Fusion for Abnormal Behaviour Detection in Activities of Daily Living," 2020 IEEE Int. Conf. Pervasive Comput. Commun. Work. PerCom Work. 2020, no. August, 2020, doi: 10.1109/PerComWorkshops48775.2020.9156249.

[6] D. Lim, C. Park, N. H. Kim, S. H. Kim, and Y. S. Yu, “Fall-Detection Algorithm Using 3-Axis Acceleration: Combination with Simple Threshold and Hidden Markov Model," J. Appl. Math., vol. 2014, 2014, doi: 10.1155/2014/896030.

[7] B. Mrazovac, B. M. Todorovic, M. Z. Bjelica, and D. Kukolj, “Reaching the next level of indoor human presence detection: An RF based solution," in 2013 11th International Conference on Telecommunications in Modern Satellite, Cable and Broadcasting Services, TELSIKS 2013, 2013, vol. 1, pp. 297-300, doi: 10.1109/TELSKS.2013.6704936.

[8] B. Lashkari, Y. Chen, and P. Musilek, “Energy management for smart homes-state of the art," Appl. Sci., vol. 9, no. 17, 2019, doi: 10.3390/app9173459.

[9] R. C. Conceição, M. O'Halloran, M. Glavin, and E. Jones, “Antenna configurations for Ultra Wide Band radar detection of breast cancer," in Progress in Biomedical Optics and Imaging - Proceedings of SPIE, 2009, vol. 7169, doi: 10.1117/12.808253.

[10] H. Medjahed, D. Istrate, J. Boudy, and B. Dorizzi, “Human activities of daily living recognition using fuzzy logic for elderly home monitoring," in IEEE International Conference on Fuzzy Systems, 2009, pp. 2001-2006, doi: 10.1109/FUZZY.2009.5277257.

[11] M. Garcia-Constantino, A. Konios, and C. Nugent, "Modelling Activities of Daily Living with Petri nets," 2018 IEEE Int. Conf. Pervasive Comput. Commun. Work., no. i, pp. 866-871, 2018, doi: 10.1109/PERCOMW.2018.8480225.

[12] T. Huang, Z. Chen, F. Xia, C. Jin, and L. Li, “A practical localization algorithm based on wireless sensor networks," Proc. 2010 IEEE/ACM Int. Conf. Green Comput. Commun. GreenCom 2010, 2010 IEEE/ACM Int. Conf. Cyber, Phys. Soc. Comput. CPSCom 2010, pp. 50-54, 2010, doi: 10.1109/GreenCom-CPSCom.2010.41.

[13] A. K. Paul and T. Sato, "Localization in wireless sensor networks: A survey on algorithms, measurement techniques, applications and challenges," J. Sens. Actuator Networks, vol. 6, no. 4, 2017, doi: 10.3390/jsan6040024.

[14] K. Daniel and C. Wietfeld, "Using Public Network Infrastructures for UAV Remote Sensing in Civilian Security Operations.," Homel. Secur. Aff., vol. 7, pp. 1-9, 2011, [Online]. Available: http://search.ebscohost.com/login.aspx?direct=true\&db=tsh\&AN=91631746\&site=ehost-live.

[15] T. O. Oshin, S. Poslad, and A. Ma, "Improving the energy-efficiency of GPS based location sensing smartphone applications," Proc. 11th IEEE Int. Conf. Trust. Secur. Priv. Comput. Commun. Trust. - 11th IEEE Int. Conf. Ubiquitous Comput. Commun. IUCC2012, pp. 1698-1705, 2012, doi: 10.1109/TrustCom.2012.184.

[16] Z. Farid, R. Nordin, and M. Ismail, "Recent advances in wireless indoor localization techniques and system," J. Comput. Networks Commun., vol. 2013, 2013, doi: 10.1155/2013/185138. 
[17] Z. Chen, F. Xia, T. Huang, F. Bu, and H. Wang, “A localization method for the Internet of Things,” J. Supercomput., vol. 63, no. 3, pp. 657-674, 2013, doi: 10.1007/s11227-011-0693-2.

[18] F. Khelifi, A. Bradai, A. Benslimane, P. Rawat, and M. Atri, “A Survey of Localization Systems in Internet of Things," Mob. Networks Appl., vol. 24, no. 3, pp. 761-785, 2019, doi: 10.1007/s11036-018-1090-3.

[19] L. Yang, Q. Lin, X. Li, T. Liu, and Y. Liu, "See through walls with COTS RFID system!," Proc. Annu. Int. Conf. Mob. Comput. Networking, MOBICOM, vol. 2015-Septe, pp. 487-499, 2015, doi: 10.1145/2789168.2790100.

[20] S. Sen, B. Radunovic, R. R. Choudhury, and T. Minka, “You are facing the Mona Lisa," p. 183, 2012, doi: $10.1145 / 2307636.2307654$.

[21] W. Wang, A. X. Liu, and M. Shahzad, “Gait recognition using WiFi signals,” UbiComp 2016 - Proc. 2016 ACM Int. Jt. Conf. Pervasive Ubiquitous Comput., pp. 363-373, 2016, doi: 10.1145/2971648.2971670.

[22] S. H. Fang, C. H. Wang, T. Y. Huang, C. H. Yang, and Y. S. Chen, “An enhanced ZigBee indoor positioning system with an ensemble approach," IEEE Commun. Lett., vol. 16, no. 4, pp. 564-567, 2012, doi: 10.1109/LCOMM.2012.022112.120131.

[23] L. Zwirello, T. Schipper, M. Harter, and T. Zwick, “UWB localization system for indoor applications: Concept, realization and analysis," J. Electr. Comput. Eng., vol. 2012, 2012, doi: 10.1155/2012/849638.

[24] M. Marek, "Practical application of coefficient of variation," Volver al Indice, vol. 1, no. 4, pp. 25-33, 2015.

[25] N. E. Elhady and J. Provost, "A systematic survey on sensor failure detection and fault-tolerance in ambient assisted living," Sensors (Switzerland), vol. 18, no. 7, 2018, doi: 10.3390/s18071991.

[26] Synnott J., Rafferty J., Nugent C., “Detection of workplace sedentary behavior using thermal sensors," Proc. Annu. Int. Conf. IEEE Eng. Med. Biol. Soc. EMBS, vol. 2016-Octob, pp. 5413-5416, 2016, doi: 10.1109/EMBC.2016.7591951. 